\title{
Trigeminal neuralgia: a plea for microvascular decompression as the first surgical option. Anatomy should prevail
}

\author{
Marc Sindou
}

Received: 20 August 2009/Accepted: 22 August 2009/Published online: 15 September 2009

(C) Springer-Verlag 2009

\begin{abstract}
Arguments are given to plead why microvascular decompression should be the first surgical option to treat primary trigeminal neuralgias resistant to anticonvulsants.
\end{abstract}

Keywords Trigeminal neuralgia - Vascular compression . Microvascular decompression · Percutaneous techniques · Radiosurgery $\cdot$ Pain

\section{Introduction}

Trigeminal neuralgia (TN) has been one-if not the firstof the major neurosurgical concerns since the speciality emerged from neurology and general surgery at the very beginning of the twentieth century. Retrogasserian neurotomy through the subtemporal approach by Frazier [29], juxtapontine rhizotomy via a retromastoid craniotomy by Dandy [4] and electrocoagulation of the Gasserian ganglion using the percutaneous transoval route of Hartel by Kirschner [12] were the first effective treatments long before anticonvulsants were revealed to be effective [2, 3].

Then, over the past decades, a number of various surgical procedures were developed with the goal of being selective to avoid (deafferentation) painful/dysesthetic phenomena, known as anesthesia dolorosa. Among these various procedures, the most popular ones were:

\footnotetext{
M. Sindou $(\bowtie)$

Department of Neurosurgery, Hôpital Neurologique

P. Wertheimer, Université Claude-Bernard of Lyon,

Groupement Hospitalier Est-59 boulevard Pinel,

69003 Lyon, France

e-mail: marc.sindou@chu-lyon.fr
}

gangliolysis with glycerol injection into the cistern of Meckel's cave [7], percutaneous R-F thermorhyzotomy [30], balloon compression of the gasserian ganglion [15] and radiosurgery targeting the trigeminal system [14]. Regarding decompression of the trigeminal root, it took a long time until it was recognized that a majority of the socalled classical primary TNs were due to a neuro-vascular conflict and before it could be established that the most effective pathophysiological method for treatment is the microvascular decompression (MVD) procedure.

It was in 1934 that Walter Dandy published his observation about performing juxtapontine rhizotomies for TN concerning the frequent compression of the trigeminal root by a neighboring elongated artery [5]. Surprisingly enough, he did not go to the point of only freeing the root from its vascular compression. The first decompression of the trigeminal root from its offending vessel was actually performed by James Gardner in 1959 [6]. But the method was really promoted by Peter Jannetta in his famous 1967 publication [8]. In his paper the trigeminal root was approached via the subtemporal-transtentorial route. The tribute of advocating the easier and safer retro-mastoid approach must be paid to Jules Hardy [19]. Then the method was codified using the microsurgical techniques by Jannetta [9] and popularized by a large number of publications.

This abundance of techniques (see the literature review in [24]) on the one hand offers patients the opportunity to benefit from an effective surgical solution, and if the first operation fails, allows them to have recourse to other different methods. But on the other hand, this variety may expose the patient to being more dependent on the surgeon's past training and on the local facilities than on a rigorous anatomical-pathological rationale. 


\section{The neurosurgical armamentarium}

The open sectioning methods, although nowadays performed safely under the microscope, have been almost totally replaced by percutaneous techniques, because the latter may achieve approximately the same neurological effects without the risks of open surgery.

At present, the percutaneous techniques most frequently used are the following.

- Glycerol injection is performed worldwide because it is not expensive. The method however entails a lack of reliability, not only in terms of duration of effect, but also of degree of neurotoxicity.

- RF-Thermorhizotomy is still in use in many neurosurgical centers. If accurately performed, the technique is selective in relation to the trigger zone $[10,22,30]$. If at least a slight hypoesthesia is produced, pain relief can be long-lasting. As an example, in our series the recurrence rate after a follow-up of up to 25 years was $7 \%$. Of the patients, 95\% had a moderate to marked hypoesthesia in their pain territory, responsible for numbness, which was well tolerated in all but $1 \%$ who had a disabling anesthesia dolorosa syndrome [28]. This technique requires specific training to avoid harmful side-effects and sequelae.

- Balloon compression of the Gasserian ganglion is considered an easier procedure. The key technical point is that the balloon must be inflated precisely inside Meckel's cave. According to the literature review, the method yields few side-effects and little hypoesthesia [11]. However, the effects are not topographically specific, and the recurrence rate is relatively high.

Stereotactic radiosurgery (RS), during the last decade, has become very popular for treating $\mathrm{TN}$ even as a first option because of its minimal invasiveness and low rate of reported complications. However, some important concerns remain. Delay in obtaining pain relief for a few weeks or even months is not that serious a problem, except perhaps for the few patients who come to surgery with intense crises. The main concern is that RS may induce delayed post-radiation lesions. Is this underestimated? Long-term data are lacking, and reports are controversial. Further, the quality of pain relief is not uniformly estimated by authors; in some publications results are classified as good or excellent even though patients are maintained on medications long after RS has been administered. That being said, the percentages of pain-free patients having had RS (namely Gamma-knife) vary from
$21.8 \%$ to $88.9 \%$ after average follow-ups ranging from 10 to 60 months. Hypoesthesia as a side-effect is reported at $0 \%$ to $54 \%$ according to the series [20]. Analysis of the literature shows that obtention of pain relief and its duration are dependent on irradiation intensity; 90 Gy is the value commonly assumed to be effective $[16,18,21]$. It has also been noted that the degree of hypoesthesia is proportional to intensity. Also, the duration of pain relief positively correlates with the presence of a postoperative hypoesthesia, and consequently of some degree of numbness. For these reasons, Pollock, who several times gave positive but also critical evaluations of the RS method, estimates that RS for TN should be considered among the lesioning techniques [16, 17].

Microvascular decompression (MVD) is a conservative method aiming at decompressing the offending $\operatorname{vessel(s)~and~also~at~freeing~the~entire~root-from~}$ Meckel's cave to the trigeminal root entry zone-from all arachnoid adhesions [27]. Importantly, in around one-third of cases there are several compressive vessels in the same patient [23].

According to the studies that include the series with a minimal averaged follow-up of 5 years and having had a Kaplan-Meier analysis [31], MVD provides the highest rate of cure. In the largest published series, the one including 1,185 patients operated on by Jannetta, $70 \%$ of the patients had an excellent result 10 years after surgery [1]. Similarly, in our series of 947 patients, of whom 362 had KM analysis, the probability of cure (i.e., no pain and no medication) was $74 \%$ at 15 years of follow-up [25].

In addition, study of prognostic factors in our series demonstrated that the presence of a marked vascular compression at surgery was correlated with a higher than $90 \%$ success rate at 15 years of FU. Conversely when the vessel was only in contact, without any visible indentation, with the root, the long-term cure rate was only $60 \%$ [26].

The presence of a NVC, the type of causative vessel and the degree of compression to the root can be predicated by imaging as demonstrated in a recent publication from our group [13].

\section{The anatomical-imaging bases}

In our opinion the surgical strategy has to be essentially and primarily based on the anatomical-pathological data, which can be infered from patients' individual imaging.

MRI exploration must include not only standard images to identify so-called symptomatic TNs that require specific treatment, but also high resolution sequences 
to detect and characterize the causative compressive vessel(s). This complementary exploration comprises the following sequences.

(1) A 3D-T2 high-resolution (i.e., millimetric) sequence allows obtaining fine and well-balanced images, with the CSF in intense hypersignal and the other structures of the cerebello-pontine angle in hyposignal, achieving a cisternography-like exploration. The limit is the absence of differentiation of signals between vessels and nerves; therefore, sequences showing vessels in hypersignal should be added.

(2) 3D-TOF (time of flight) MRA, if performed with a presaturation filter, visualizes (only) the highflow vessels, namely the arteries.

(3) A 3D-T1-weighted sequence with gadolinium is able to depict all vascular structures, both arteries and veins; they are enhaced by contrast medium.

Combination of the latter two sequences is indispensable to differentiate veins from arteries.

In a recent prospective study comparing the imaging data and the findings at surgery with a blinded protocol, in 100 consecutive cases, we found that the sensitivity of MRI was $96.7 \%$ for detecting neurovascular conflicts and specifity $100 \%$. Further, the MRI value for predicting the type of the responsible vessel(s) was $88 \%$, the location along and the site around the root $85.7 \%$ and $84.6 \%$, respectively, and - most important-the degree of compression $84.6 \%$; the $p$ value for all these features was $<0.01$ [13].

\section{Conclusions}

As seen above, complete MRI exploration is of extreme practical importance; it gives the surgeon the necessary anatomical bases for indicating surgery and choosing the more appropriate procedure.

It is now well accepted that vascular compressions are the origin of a large majority of the so-called primary TNs. This has been documented through a number of publications and validated by the solidity of the percentages of permanent cures obtained by pure MVD surgery [27] in the main series (quoted in [31]). Therefore, we do think that MVD - because of its conservative nature - should be considered as the first surgical choice when the patient's conditions are satisfactory. In experienced hands, complications are uncommon.

Percutaneous lesioning techniques or radiosurgery is indicated in patients in whom imaging does not provide clear-cut evidence of a vascular compression. They constitute a providential recourse when patients are in precarious conditions. Absence of hypoesthesia in the painful area correlates with a high probability of early recurrence; contrarily, a marked hypoesthesia entails the risk of anesthesia dolorosa, and if the cornea is affected, the risk of keratitis.

MVD being a conservative method, it can be considered the gold stantard. In the field of surgery, history shows that anatomical-based operations ultimately prevail, provided they are performed by well-trained teams.

\section{References}

1. Barker FG, Jannetta PJ, Bissonette DJ, Larkins MV, Jho HC (1996) The long-term outcome of microvascular decompression for trigeminal neuralgia. N Engl J Med 334:1077-1083

2. Bergouignan M (1942) Cures heureuses de névralgies faciales essentielles par le diphényl-hydantoinate de soude. Rev Laryngol Oto Rhinol (Bordeaux) 63:34-41

3. Blom S (1962) Trigeminal neuralgia: its treatment with a new anticonvulsant drug (G-32883). Lancet 1:839-840

4. Dandy WE (1929) Operation for cure of tic douloureux; partial section of the sensory root at the pons. Arch Surg 18:687-734

5. Dandy WE (1934) Concerning the cause of trigeminal neuralgia. Am J Surg 24:447-455

6. Gardner WJ, Miklos MV (1959) Response of trigeminal neuralgia to decompression of sensory root. Discussion of cause of trigeminal neuralgia. JAMA 170:1773-1776

7. Hakanson S (1981) Trigeminal neuralgia treated by the injection of glycerol into the trigeminal cistern. Neurosurgery 9:638-646

8. Jannetta PJ (1967) Arterial compression of the trigeminal nerve at the pons in patients with trigeminal neuralgia. J Neurosurg 26:159-162

9. Jannetta PJ (1976) Microsurgical approach to the trigeminal nerve for tic douloureux. In: Krayenbuhl H, Maspes PE, Sweet WH (eds) Progress in Neurological Surgery, vol.7. Pain-Its neurosurgical management. Karger S, Basel, pp 180-200

10. Kanpolat Y, Savas A, Bekar A, Berk C (2001) Percutaneous controlled radiofrequency trigeminal rhizotomy for the treatment of idiopathic trigeminal neuralgia: 25 -year experience with 1,600 patients. Neurosurgery 48:524-532

11. Keravel Y, Gaston A, de Andrade C, Mencattini G, Le Guerinel C (2009) Traitement de la névralgie trigéminale par la compression par ballon. Neurochirurgie 55:197-202

12. Kirschner M (1932) Zur Elektrocoagulation des Ganglion Gasseri. Zentrabl Chir 59:2841-2843

13. Leal PRL, Froment JC, Sindou M (2009) Valeur predictive de l'IRM pour la détection et la caractérisation de la compression vasculaire dans les syndromes d'hyperactivité des nerfs crâniens (trijumeau et facial). Neurochirurgie 55:174-180

14. Leksell L (1971) Stereotaxic radiosurgery in trigeminal neuralgia. Acta Chir Scan 137:311-314

15. Mullan S, Lichtor T (1983) Percutaneous microcompression of the trigeminal ganglion for trigeminal neuralgia. J Neurosurg 59:1007-1012

16. Pollock BE (2006) Radiosurgery for trigeminal neuralgia: is sensory disturbance required for pain relief? J Neurosurg (suppl) 105:103-106

17. Pollock BE, Phuong LK, Foote RL, Stafford SL, Gorman DA (2001) High-dose trigeminal neuralgia radiosurgery associated with increased risk of trigeminal nerve dysfunction. Neurosurgery 49:58-64 
18. Pollock BE, Phuong LK, Gorman DA, Poote RL, Stafford SL (2002) Steteotactic radiosurgery for idiopathic trigeminal neuralgia. J Neurosurg 97:347-353

19. Provost J, Hardy J (1970) Microchirurgie du trijumeau: anatomie fonctionnelle. Neurochirurgie 16:459-470

20. Regis J, Arkha Y, Yomo S, Murata N, Roussel P, Donnet A, Peragut JC (2009) La radiochirurgie dans le traitement de la névralgie trigéminale: résultats à long terme et influence des nuances techniques. Neurochirurgie 55:213-222

21. Regis J, Metellus P, Hayashi M, Roussel P, Donnet A, Bille-Turc (2006). Prospective controlled trial of gamma-knife surgery for essential trigeminal neuralgia. J Neurosurg 104:913-924

22. Sindou M (1999) Neurophysiological navigation in the trigeminal nerve: use of masticatory responses and facial motor responses evoked by electrical stimulation of the trigeminal rootlest for RFthermorhyzotomy guidance. Stereot Funct Neurosurgery 73:117121

23. Sindou M, Howeidy T, Acevedo G (2002) Anatomical observations during microvascular decompression for idiopathic trigeminal neuralgia (with correlations between topography of pain and site of the neurovascular conflicts). Prospective study in a series of 579 patients. Acta Neurochirurgica 144:1-13

24. Sindou M, Kéravel Y et al (2009) Neurochirurgie fonctionnelle dans les syndromes d'hyperactivité des nerfs craniens. Rapport à la Société de Neurochirurgie de Langue Française. Neurochirurgie 55:75-292
25. Sindou M, Leston J, Howeidy T, Decullier E, Chapuis F (2006) Microvascular decompression for primary trigeminal neuralgia (typical or atypical). Long-term effectiveness on pain; prospective study with survival analysis in a consecutive series of 362 patients. Acta Neurochir 148:1235-1245

26. Sindou M, Leston JM, Decullier E, Chapuis F (2007) Microvascular decompression for primary trigeminal neuralgia: long-term effectiveness and prognostic factors in a series of 362 consecutive patients with clearcut neurovascular conflicts who underwent pure decompression. J Neurosurg 107:1144-1153

27. Sindou M, Leston JM, Decullier E, Chapuis F (2008) Microvascular decompression for trigeminal neuralgia: the importance of a non compressive technique-Kaplan-Meier analysis in a consecutive series of 330 patients. Neurosurgery 63:341-351

28. Sindou M, Tatli M (2009) Traitement de la névralgie trigéminale par thermorhizotomie. Neurochirurgie 55:203-210

29. Spiller WG, Frazier CH (1901) The division of the sensory root of the trigeminus for the relief of tic douloureux; an experimental, pathological and clinical study, with a preliminary report of one surgically successful case. Philadelphia Med J 8:1039-1049

30. Sweet WH, Wepsic JG (1974) Controlled thermocoagulation of trigeminal ganglion and rootlets for differential destruction of pain fibers; part I: trigeminal neuralgia. J Neurosurg 40:143-156

31. Tatli M, Satici O, Kanpolat Y, Sindou M (2008) Various surgical modalities for trigeminal neuralgia: literature study of respective long-term outcomes. Acta Neurochirurgica 150:243-255 